\title{
Automated Measurement of Attitudes Towards Social Distancing Using Social Media: A COVID-19 Case Study
}

\begin{abstract}
The COVID-19 outbreak has focused attention on the use of social distancing as the primary defence against community infection, yet forcing social animals to maintain that physical distance has presented significant challenges for health authorities and law enforcement. Anecdotal media reports suggest widespread dissatisfaction with social distancing as a policy, yet there is little prior work aimed at measuring community acceptance of social distancing. In this paper, we propose a new approach to measuring attitudes towards social distancing by using social media and sentiment analysis. Over a 4 month period, we found $82.5 \%$ of tweets were in favour of social distancing. The results indicate a widespread acceptance of social distancing in the community. We conclude by examining options for estimating the optimal (minimal) social distance required at scale, and the implications for securing widespread community support and acceptance of public health messaging.
\end{abstract}

A. S. M. Kayes, La Trobe University, a.kayes@latrobe.edu.au

Md. Saiful Islam, Griffith University, saiful.islam@griffith.edu.au

Paul A. Watters, La Trobe University, p.watters@latrobe.edu.au

Alex Ng, La Trobe University, alex.ng@latrobe.edu.au

Humayun Kayesh, Griffith University, humayun.kayesh@griffithuni.edu.au

\section{Contents}

Introduction

Coronavirus Infections and Consequences Across Australia

Proposed Model for Measuring Attitudes towards Social Distancing

Results and Data Analysis

Discussion

Conclusion 


\section{Introduction}

The outbreak of COVID-19 has had a significant impact on the core dimensions of daily life for the global population (COVID-19 Case, 2020). As an advanced economy in the Asia-Pacific, Australia has been no exception. Media reports suggest that the first COVID-19 case was confirmed on January 25, 2020 in Australia and the confirmed cases appeared to be doubling in every three days, however, formal advice from the state Department of Health and Human Services (DHHS) on social distancing (Glass et al, 2006) was only announced on March 12, 2020 (COVID-19 Double, 2020). Based on DHHS guidelines, a requirement to ensure a physical distance of 1.5 meters between people was mandated as the primary social distancing measure (DHHS, 2020). According to a message from the Australian government and Chief Medical Officer (VIC Govt., 2020), achieving widespread compliance with social distancing measures over the course of at least the next six months will "flatten the curve" (Rhoades et al., 2020), thereby reducing the burden on over-stretched public health facilities.

However, there is a direct conflict between the public health requirement to "socially distance" and the natural human desire for social interaction (Cohen, 2004). Classic attachment theory suggests that humans (and other primates) have an innate and natural desire for social and physical contact, that begins from early life (Bowlby, 1979). Child primates denied physical contact with their mothers will go to extreme lengths to achieve that contact, even engaging in self-injurious behaviour. As Bowlby himself said (Bowlby, 1958), "separation experiences are pathogenic". Anecdotal reports from the current crisis see a range of pathological and stress-based responses to enforced social isolation, from aggression in supermarkets over toilet tissue and spitting with the intent to infect, through to breaking laws to breach social distancing limits. Furthermore, the mental health consequences of social isolation (Harlow et al., 1965) cannot be ignored in the quest to suppress infectious diseases: there is significant comorbidity of anxiety and depression in Australia, with elderly people being chronically affected (Almeida et al, 2012) - ironically, social distancing is meant to protect elderly people who are also at higher risk of contracting COVID-19 (Porcheddu et al., 2020).

There are many active ways that people can be affected by coronavirus patients. Based on the COVID-19 worldwide survey (COVID-19, 2020), people who have been admitted to hospitals for emergency treatments, are affected by the coronavirus patients, and the consequences have appeared as severe cases often resulting in death. Though the primary goal of social distancing measures is to slow the curve against coronavirus infections, another important issue is the COVID-19 test delays that people who suspect 
they have coronavirus. To achieve the greater good of social distancing, the non-infected population are compelled to behave in a way which defies their natural desire to socialise (Stewart et al., 2015). On the other hand, non-infected individuals will benefit potentially from the overall reduction in the spread of the disease, since eradication or suppression will reduce their own chances of being infected. The selfish (and selfless) responses to infectious diseases have been examined previously in relation to HIV (Blechner, 1993) and ebola (Falade \& Coultas, 2017), yet there has been no previous research specifically investigating attitudes towards social distancing. Yet the success of social distancing as an infection prevention strategy requires widespread social acceptance and adoption. How do we resolve this circularity?

One approach that we explore in this paper is to identify, model and measure all of the relevant parameters we should consider to solve the social distancing problem. In mathematical terms, we need to maximise social distancing to the extent that is medically necessary to suppress viral transmission, while minimising the psychological distress and harm that is caused by social distancing and social isolation.

According to the Australian Government Department of Health (DoH, 2020), COVID-19 is most likely spreading in any of the following three possible ways: (1) direct close contact with a confirmed case; (2) close contact with the droplets of a carrier of the virus who coughs or sneezes; or (3) touching objects or surfaces contaminated with COVID-19 virus dispersed by a carrier of the virus, and then touching the mouth or rubbing the nose. Social distancing (DHHS, 2020) is one of the crucial ways to reduce the peak of the COVID-19 pandemic, where different physical distancing restrictions are associated with such measurement to protect against COVID-19, like no gatherings of more than 2 persons, stay at home and only go out if it is absolutely essential, keep 1.5 metres away from others, and avoid physical greetings such as handshaking, hugs and kisses.

When we speak to the main root cause of spreading coronavirus cases, we refer to the social distancing parameters as a preventive measure (Gillis et al., 2000). However, the role of social media against spreading coronavirus is another important parameter to be considered as a social distancing measure. This is really another great challenge to identify the passive ways to protect people against affecting viruses. There is a great need for using as much data from social media platforms such as Twitter data about people's attitudes - people's attitudes towards COVID-19, including test delays and influencers' hashtags related to government messages, and panic related to the rising curve of virus cases. 
This research proposes a solution of ensuring an appropriate social distancing measure and protecting against spreading coronavirus infections. We provide some preliminary explanations for why people may be so willing to subvert critical controls in social distancing, intentionally or lack of awareness - in cognate attempts to reduce illness through messaging, such as tobacco control, awareness and messaging have been effective (Chapman, 2008). Without awareness, the vast majority of people have no idea about the preventive parameters that can contribute to measuring social distance, notwithstanding the role of the media in promoting government hegemony in relation to public health (Wallack \& Dorfman, 1992). In this paper, we use social media data about people's sentiments and experts' predictions, especially from Twitter (Layton et al., 2010). We also use real COVID-19 datasets about infected people along with total cases by states and territories across Australia, for instance to know about how frequently symptoms are growing and varying in different states.

The first step in exploring behaviours is to explore the attitudes that give rise to the behaviour. One way to do this would be to use self-report measures such as polls or questionnaires. Yet we know that such measures have innate biases, such as self-report bias - only those persons with a particular interest are likely to respond. In order to address these biases, observational techniques to measure attitudes have been proposed. Previous research has suggested using Social Media Sentiment Analysis (SMSA) to measure attitudes at scale; Prichard et al (2015) developed an approach to measuring the sentiments expressed in tweets and other types of social media to answer complex questions about public attitudes towards crime and criminals. The results - matching findings from attitudinal surveys (Prichard et al., 2016) - suggest that the population has quite sophisticated and complex attitudes towards seemingly simple questions about crime and criminals.

We propose to use SMSA to explore public attitudes towards social distancing measures, as the first step in developing a more sophisticated model of optimal social distancing. Twitter has a long history of being used for surveillance of infectious disease outbreaks (eg, Signorini et al, 2011): can we use tweets and other social media data to better understand public sentiment towards social control measures that are intended to reduce infection? Can we predict what social attitudes to new and stricter measures are likely to be, in the same way that tweets can be used to predict disease outbreaks (St Louis \& Zorlu, 2012)? A sophisticated model that can describe and predict public sentiment will be extremely useful to lawmakers and public policy officials in the coming months and years, we assert. 


\section{Coronavirus Infections and Consequences Across Australia}

Based on the report on 16 March 2020 (COVID-19 Double, 2020), Australian COVID-19 cases appeared to be doubling in every three days, though the restrictions on social distancing are already taken into account on March 12, 2020. The Australian prime minister has announced another social distancing measure on 29 March (COVID-19 Restrictions, 2020) along with a stage- 3 restrictions on gatherings, like more than 2 persons are not allowed to gather physically unless they are household residents, even if not in the outdoor spaces like backyards.

From a purely descriptive perspective, we consider COVID-19 cases across Australia from 20 February to 27 March 2020. Figure 1 demonstrates the 2-timeline of COVID-19 cases across Australia: (a) the time duration until when the social distancing restrictions taken into account, on the 12 March 2020 (DHHS, 2020), and (b) another time duration until declaring a clear guideline on non-essential closures on March 27, 2020 (VIC Govt., 2020).

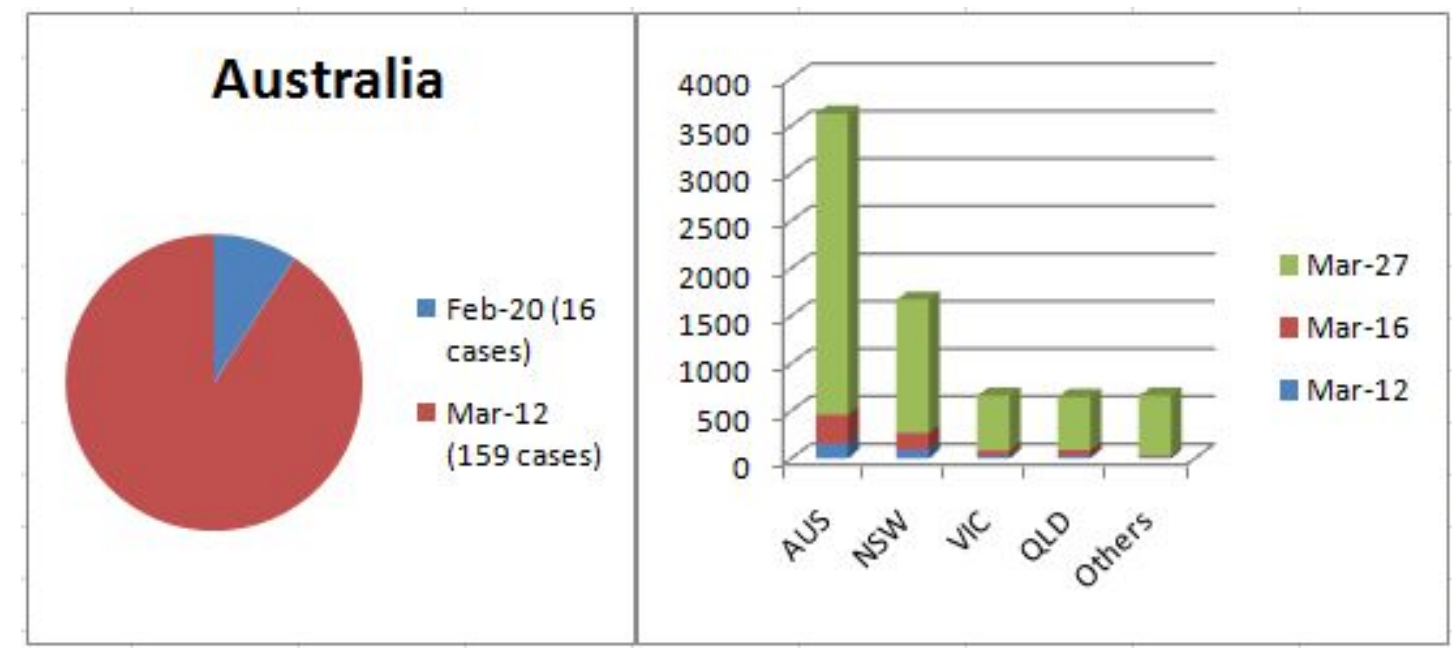

(a)

(b)

Figure 1: Australian COVID-19 cases from 20 February 2020 27 March 2020: (a) Number of cases before Social Distancing Measures taken into account, (b) Number of cases after Social Distancing Measures taken into account.

The numbers in Figure 1 show Australian COVID-19 cases that are on a similar trajectory like a global pandemic with an exponential growth. Both timelines share a significant number of virus cases considering before and after social distancing restrictions taken into place. The numbers reveal the dilemma of achieving social distancing when humans are innately social creatures. We observed the number of 
confirmed cases increased from 159 on March-12 (date declared social distancing) to over 3500 on March-27, after 14 days of enforcing "social distancing".

In the literature, there are studies in school practices to promote social distancing in K-12 schools (Uscher-Pines, et al., 2018) and advice on other measures for response to an influenza pandemic (Rashid, et al., 2015). Those studies show lacking information available to schools to develop policies and procedures on social distancing, and the effectiveness of social distancing requires high restrictions and at a critical period of within ten days before the peak of a pandemic. Other landmark studies (e.g., Maharaj \& Kleczkowski, 2012) have focused on modelling the purely economic outcomes associated with different social distancing strategies, completely ignoring the social impact (including compliance) driven by social attitudes.

We argue that those studies are inconclusive and vital factors are missing that have impacted the effectiveness of social distancing in slowing down the spread of COVID-19 cases.

\section{Proposed Model for Measuring Attitudes towards Social Distancing}

One of the biggest challenges of implementing a defense mechanism against a pandemic is to ensure public participation and acceptance of any mechanism proposed by the authority (Young, 2013). Therefore, understanding how people perceive the pandemic and the sentiment towards any mechanism is important in relation to successful implementation of a defense mechanism against a pandemic. Social media is a great source of public opinions, which has been explored to understand citizen reactions and propagation of Ebola related information in the past (Tran et al., 2016).

In this paper, we have proposed a data driven automated measurement approach to measuring social attitudes towards any defence mechanism to respond to a pandemic such as "social distancing" in case of COVID-19 outbreak in Australia. Our measurement model is a transfer learning (Delvin et al., 2019) based deep twitter sentiment detection model. Figure 2 illustrates a schematic diagram of our proposed model. The central to our model is social media data such as tweets. We have used publicly available sentiment analysis training corpus ${ }^{1}$ to train our model and then, applied the trained model to assess the sentiments of the tweets collected during the period Dec 01, 2019 Mar 30, 2020 with hashtag \#coronavirus within Australia.

\footnotetext{
${ }^{1}$ http://thinknook.com/twitter-sentiment-analysis-training-corpus-dataset-2012-09-22/
} 


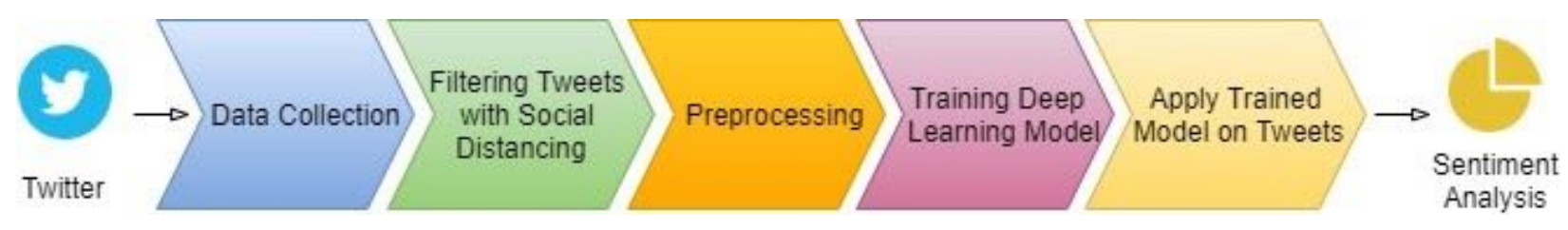

Figure 2: Schematic diagram of the twitter sentiment analysis model

\section{Results and Data Analysis}

We have collected a total of $100 \mathrm{~K}$ tweets with hashtag \#coronavirus within Australia. Out of these $100 \mathrm{~K}$ tweets, 3,076 tweets contain the keyword "social distancing" and/or hashtag \#socialdistancing. Figure 3 illustrates a wordcloud prepared from this twitter dataset. We find that the most prominent word in the wordcloud is "social distancing", which suggests that this was the most discussed topic during the period.

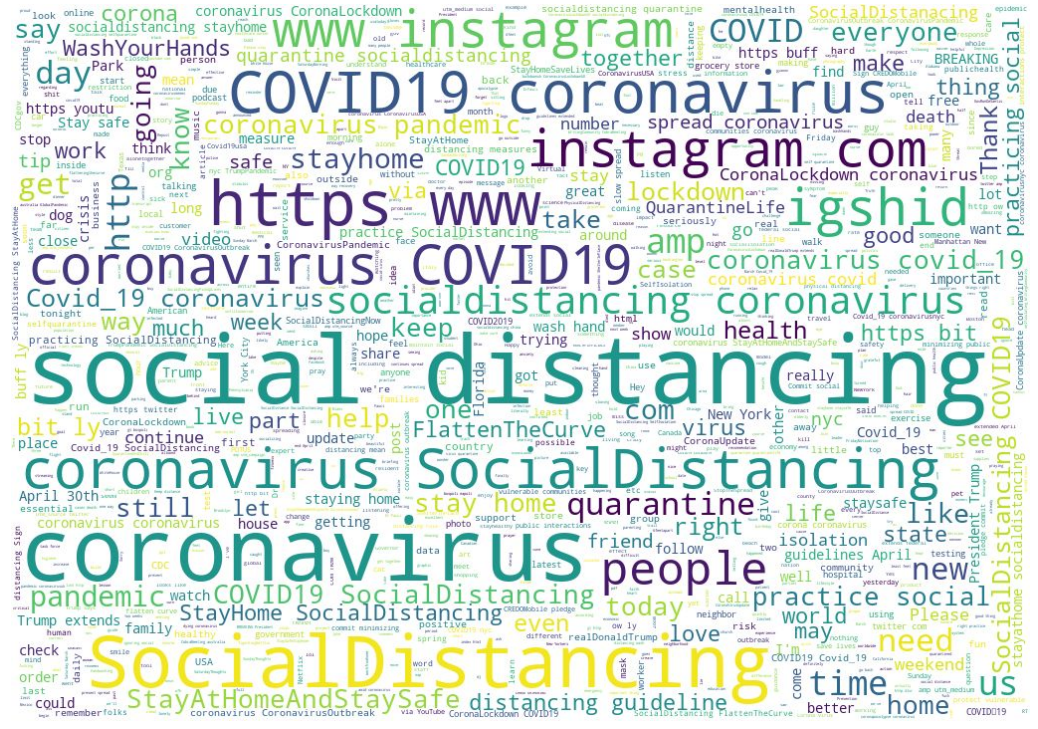

Figure 3: Word cloud of downloaded tweets, where "social distancing" is the most discussed topic period Dec 01, 2019 Mar 30, 2020 in Australia

To further understand the topics people mentioned most in tweets during the period, we have extracted the top 15 uni-grams, bi-grams and tri-grams from the collected tweets. The uni-grams, bi-grams and tri-grams correspond to one-word, two-word, and three-word phrases used in tweets. Figures 4 (a), (b) and (c) show that the most used phrases include: 'coronavirus', 'social distancing' and 'practicing social distancing'. Additionally, 'covid19', 'quarantine' and 'pandemic' were among the top 15 single-word phrases during the period. Figure $4(d)$ focuses on the top 15 hashtags 
used during the period ${ }^{2}$. The figure suggests that the most used hashtags during this period is '\#covid19', which was mentioned in more than $25 \mathrm{~K}$ tweets out of $100 \mathrm{~K}$ tweets used in our analysis. The frequently used phrases like 'practice social distancing' and the common hashtags such as '\#stayhome' and '\#stayhomestaysafe' suggest that a good number of people supports the idea of social distancing.

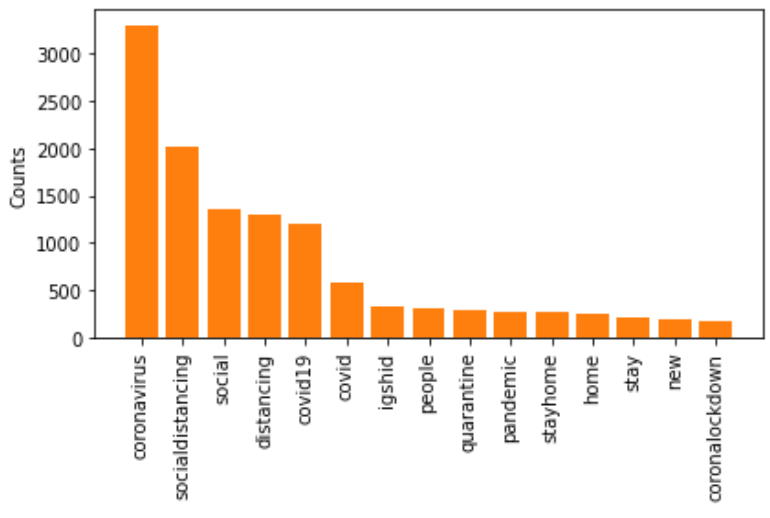

(a)Top 15 uni-grams

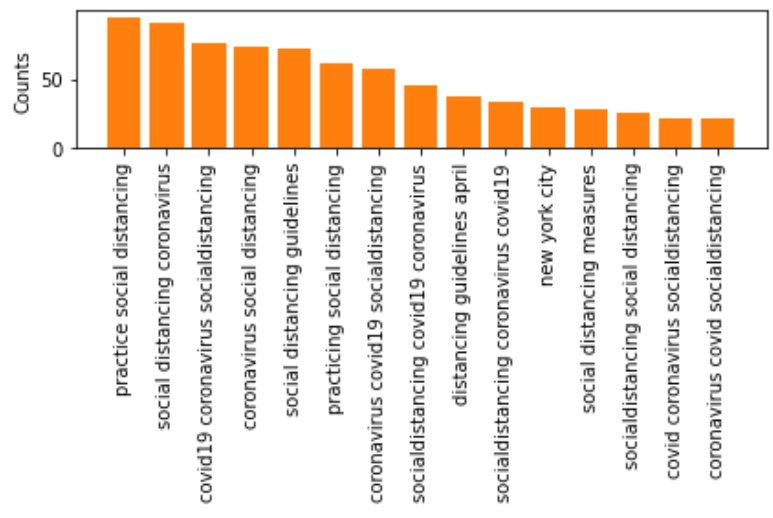

(c) Top 15 tri-grams

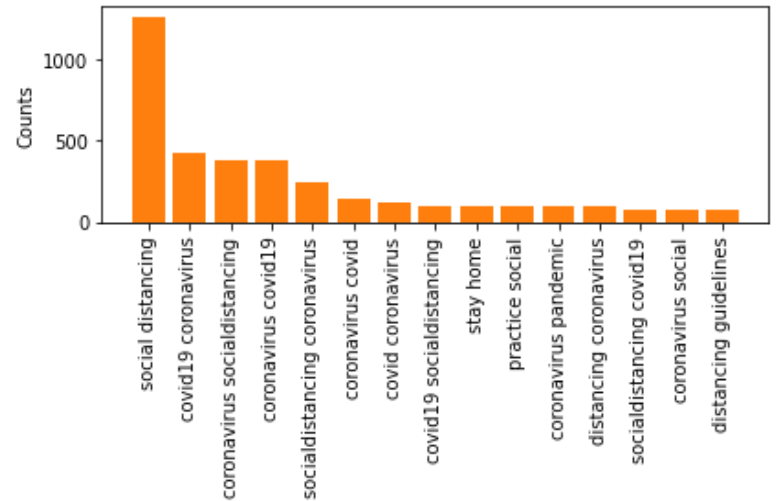

(b) Top 15 bi-grams

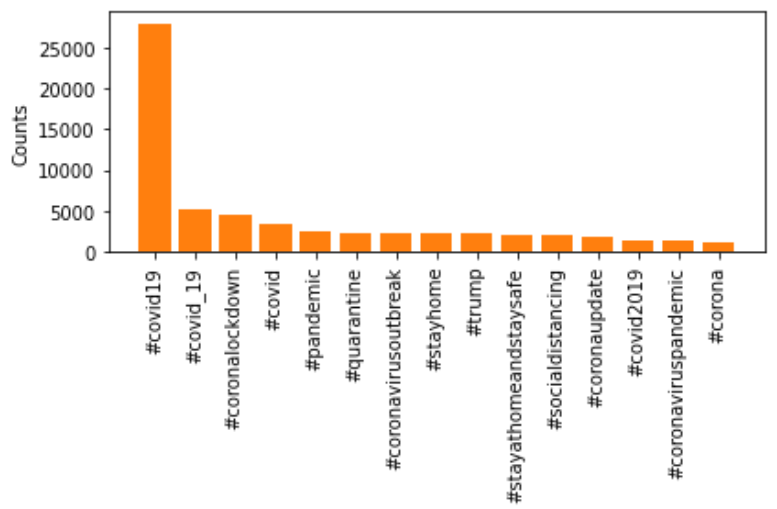

(d)Top 15 hashtags other than \#coronavirus

Figure 4: The most frequent word phrases: (a) one-word, (b) two-word, and (c) three-word phrases, respectively; and (d) the most used hashtags (other than \#coronavirus) mentioned in tweets during period Dec 01, 2019 Mar 30, 2020 in Australia

To further analyse peoples' perception on "social distancing", we have fine-tuned and trained our sentiment detection model (as illustrated in Figure 2) on the collected tweets. We use $8 \mathrm{~K}$ tweets for training and validation and $2 \mathrm{~K}$ tweets for testing our

\footnotetext{
${ }^{2}$ Here, we exclude the hashtag \#coronavirus as we use this hashtag as the search key for collecting the tweets.
} 
model. Our model has achieved $83.70 \%$ accuracy and $81.62 \%$ f1-score on the test dataset. We then applied the trained model on the 3,076 tweets that mention "social distancing" as a hashtag and/or a simple text in the tweet. We have found that more than $80 \%$ of the tweets that talked about "social distancing" have positive sentiment as illustrated in Figure 5. This reveals the fact that social distancing as a measure to protect the community against COVID-19 have been well accepted in Australia, despite the resulting social isolation.

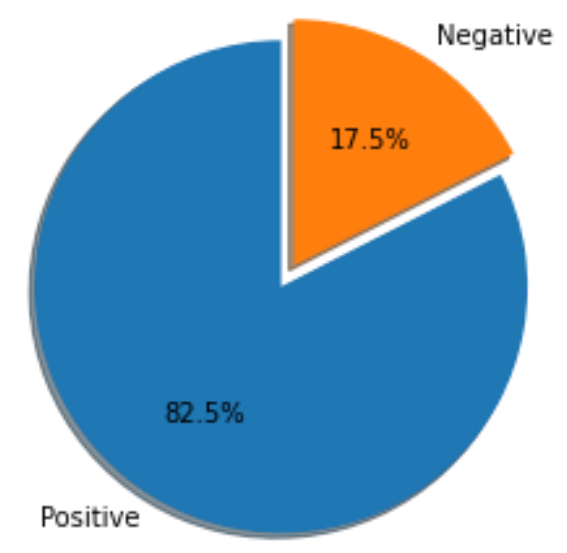

Figure 5: Sentiments (in favour/not in favour of "social distancing") of tweets tweets that mentioned social distancing during period Dec 01, 2019 Mar 30, 2020 in Australia

\section{Discussion}

At first glance, COVID-19 crisis management and communication strategies have varied wildly between countries. We believe this paper might open up new avenues of future symptomatic coronavirus research for different scientific communities, especially in predicting public attitudes towards future social controls that may become necessary. In future work, we propose to use different artificial intelligence techniques to learn about the impact of social media on the COVID-19 panic cycle of the people in a country, and to predict its trajectory. Furthermore, we will also investigate the role of messaging and chatbots in engaging concerned users at a very large scale - our current corpus of $100 \mathrm{~K}$ represents a small sample of all global discourse concerning COVID-19.

According to the statistics from the Department of Health (DoH, 2020; COVID-19 Case, 2020), there have been 3166 confirmed cases in Australia, including 367 severe and mild cases of COVID-19 as on 27 March 2020. As the situation with COVID-19 
continues to change at a rapid pace (COVID-19 Case, 2020), we can have the following two observations.

- Either the social distancing measure is not taken into account by all residents.

- Or, the parameters/metrics that are associated with such distancing are not adequate to combat spreading COVID-19 infections.

Given that we have shown widespread support for social distancing, we believe the second observation carries more weight. We now present a two-layer social distancing model for the effective COVID-19 crisis management to protect against spreading coronavirus cases. In order to prepare an appropriate crisis communication plan, we can cluster two layers of social distancing parameters (imposed" versus "derived" parameters).

- The parameters that are directly imposed by the governments.

- The parameters that can be identified through data analytics on people's sentiments.

As demonstrated in the earlier sections, the coronavirus cases are still growing rapidly though the Governments are trying to impose different restrictions on social gatherings, mostly those measures are all about physical gathering restrictions. As such, in this study we propose an approach to measuring attitudes towards social distancing so that we can separate the government-imposed physical distancing restrictions from the social gathering restrictions that can be identified from COVID-19 related social media data. In our future research, we will extend this model and propose a technical/mathematical model along with using more insights from both datasets: (i) coronavirus symptomatic data from officials, and (ii) people's sentimental data from social platforms.

The Coronavirus Mobile App is an initiative by Australian government that currently offers up-to-date advice and information on COVID-19 (Coronavirus Mobile App, 2020). It provides eight distinct features for both iOS and Android platforms, including 'symptom checker' along with a diagnostic tool from the Australian Department of Health, 'register isolation' for users in self-isolation, and so on. The coronavirus app can be extended through incorporating the social distancing model that we proposed in this paper - using a wide range of Al methods (including machine learning algorithms) so that it can provide the relevant alert-features for instance.

The data we have examined in this study are obviously not going to be able to answer the optimisation problem - we can use data and other relevant opportunities to have 
some inputs onto COVID-19, for example, we can either think through using data mining for modelling and protection of the disease spread and other hazardous consequences, or around modelling and understanding risks that are associated with coronavirus infections. However, the overarching parameters for social distancing measures that we have observed here are still worthy of analysis. The observation along with experimental results from this research can be used as our national safety measure, like locking down the specific regions/suburbs where mass gathering or panic buying is happening, that is the government-imposed social distancing measures are not appropriately taken into consideration.

\section{Conclusion}

A new social distancing model for COVID-19 has been introduced based on measuring the natural human desire for social and physical interactions by social media. We have identified, modeled and measured the relevant parameters for "social distancing": (i) the parameters that are directly imposed by the governments, like a 1.5-meter physical distancing restriction; and (ii) other parameters that are identified through data analytics, such as from the people's sentiments. Instead of government imposed parameters, what we see is that new parameters are also necessary in relation to physical distancing restrictions.

Although the proposed model is also applicable to the management of the COVID-19 crisis, it has been formulated primarily for those infected cases whose occurrences are more likely because of the lack of applying appropriate social distancing measures. We have considered not only the statistics about COVID-19 from the official announcements of local and federal governments across Australia, but also examined the survey of social media data about "social distancing".

We believe that our model and analysis outcome provide new insights into the COVID-19 outbreak by considering public reactions and sentiments towards defense mechanisms. We have also provided a foundation for data driven automated analysis of public understandings and responses that could be exercised as a useful tool in future public health crises.

\section{About the Authors}

Dr. A. S. M. Kayes is a Lecturer in Cybersecurity at La Trobe University, Australia. He currently holds a research grant from the Oceania Cybersecurity Centre (OCSC). His 
research interests include information modelling, security and access control, cloud and fog computing, loT security, ransomware detection and defence mechanism. E-mail: a [dot] kayes [at] latrobe [dot] edu [dot] au

Dr. Md. Saiful islam is a Lecturer in Big Data Analytics at Griffith University, Australia. His research interests include database usability, advanced data analytics, graph data management, machine learning and security analytics.

E-mail: saiful [dot] islam [at] griffith [dot] edu [dot] au

Paul A. Watters is Professor of Cybersecurity at La Trobe University, and leader of the Cybersecurity and Networking Research Group. Professor Watters currently holds an Australian Discovery Council grant and three grants from the Oceania Cybersecurity Centre (OCSC). His research interests lie in the human factors which underpin (or undermine) cybersecurity. He was previously a consultant to the Medical Research Council in the UK, developing groundbreaking approaches to enable secure access to public health datasets.

E-mail: $p$ [dot] watters [at] latrobe [dot] edu [dot] au

Dr. Alex $\mathbf{~ N g}$ is a Lecturer in Cybersecurity at La Trobe University, Australia. His research interests span the fields of blockchain security, blockchain-based B2B collaboration, Al-based security defence for intelligent city, malware detection and prevention mechanisms.

E-mail: alex [dot] ng [at] latrobe [dot] edu [dot] au

Humayun Kayesh is a PhD student in the Griffith School of Information and Communication Technology. His research interests include natural language processing, data analytics and deep learning.

E-mail: humayun [dot] kayesh [at] griffithuni [dot] edu [dot] au

\section{References}

Almeida, O. P., Draper, B., Pirkis, J., Snowdon, J., Lautenschlager, N. T., Byrne, G., ... \& Pfaff, J. J. (2012). Anxiety, depression, and comorbid anxiety and depression: risk factors and outcome over two years. International Psychogeriatrics, 24(10), 1622-1632.

Blechner, M. J. (1993). Psychoanalysis and HIV disease. Contemporary Psychoanalysis, 29(1), 61-80. 
Bowlby, J. (1958). The nature of the child's tie to his mother. International journal of psycho-analysis, 39, 350-373.

Bowlby, J. (1979). The Bowlby-Ainsworth attachment theory. Behavioral and Brain Sciences, 2(4), 637-638.

Chapman, S. (2008). Public health advocacy and tobacco control: making smoking history. John Wiley \& Sons.

Cohen, S. (2004). Social relationships and health. American psychologist, 59(8), 676.

Devlin, J., Chang, M., Lee, K., \& Toutanova, K. (2019). BERT: Pre-training of Deep Bidirectional Transformers for Language Understanding, arXiv preprint arXiv:1810.04805.

Falade, B. A., \& Coultas, C. J. (2017). Scientific and non-scientific information in the uptake of health information: The case of Ebola. South African Journal of Science, 113(7-8), 1-8.

Gillis, D., Yetiv, N., Gdalevich, M., Mimouni, D., Ashkenazi, I., Shpilberg, O., ... \& Shemer, J. (2000). Active versus passive immunization against hepatitis $A$ in the Israel defence forces: a cost-benefit analysis. Vaccine, 18(26), 3005-3010.

Glass, R. J., Glass, L. M., Beyeler, W. E., \& Min, H. J. (2006). Targeted social distancing designs for pandemic influenza. Emerging infectious diseases, 12(11), 1671.

Harlow, H. F., Dodsworth, R. O., \& Harlow, M. K. (1965). Total social isolation in monkeys. Proceedings of the National Academy of Sciences of the United States of America, 54(1), 90.

Layton, R., Watters, P., \& Dazeley, R. (2010, July). Authorship attribution for twitter in 140 characters or less. In 2010 Second Cybercrime and Trustworthy Computing Workshop (pp. 1-8). IEEE.

Maharaj, S., \& Kleczkowski, A. (2012). Controlling epidemic spread by social distancing: Do it well or not at all. BMC Public Health, 12(1), 679.

Porcheddu, R., Serra, C., Kelvin, D., Kelvin, N., \& Rubino, S. (2020). Similarity in Case Fatality Rates (CFR) of COVID-19/SARS-COV-2 in Italy and China. The Journal of Infection in Developing Countries, 14(02), 125-128. 
Prichard, J., Spiranovic, C., Gelb, K., Watters, P. A., \& Krone, T. (2016). Tertiary education students' attitudes to the harmfulness of viewing and distributing child pornography. Psychiatry, Psychology and Law, 23(2), 224-239.

Prichard, J., Watters, P., Krone, T., Spiranovic, C., \& Cockburn, H. (2015). Social media sentiment analysis: A new empirical tool for assessing public opinion on crime?. Current Issues in Criminal Justice, 27(2), 217-236.

Rashid, H., I. Ridda, C. King, M. Begun, H. Tekin, J. G. Wood and R. Booy (2015). "Evidence compendium and advice on social distancing and other related measures for response to an influenza pandemic", Paediatric respiratory reviews 16(2): 119-126. accessed 27 March 2020.

Rhodes, T., Lancaster, K., \& Rosengarten, M. (2020). A model society: maths, models and expertise in viral outbreaks.

Signorini, A., Segre, A. M., \& Polgreen, P. M. (2011). The use of Twitter to track levels of disease activity and public concern in the US during the influenza A H1N1 pandemic. PloS one, 6(5).

Stewart, J., Browning, C., \& Sims, J. (2015). Civic Socialising: a revealing new theory about older people's social relationships. Ageing \& Society, 35(4), 750-764.

StLouis, C., \& Zorlu, G. (2012). Can Twitter predict disease outbreaks?. Bmj, 344, e2353.

Tran, T., \& Lee, K. (2016). Understanding citizen reactions and Ebola-related information propagation on social media, IEEE/ACM International Conference on Advances in Social Networks Analysis and Mining (ASONAM). IEEE, pp. 106-111.

Young, O. R. (Ed.). (2013). Compliance \& public authority: A theory with international applications. Routledge.

Uscher-Pines, L., H. L. Schwartz, F. Ahmed, Y. Zheteyeva, E. Meza, G. Baker and A. Uzicanin (2018). School practices to promote social distancing in K-12 schools: review of influenza pandemic policies and practices, BMC public health 18(1): 406. accessed 27 March 2020.

Coronavirus Mobile App, The government's coronavirus mobile app is a solid effort, but it could do even better, published on 30 March 2020. 
http://theconversation.com/the-governments-coronavirus-mobile-app-is-a-solid-effort-but -it-could-do-even-better-135030. accessed 31 March 2020.

COVID-19 - What you need to know about coronavirus, 2020.

https://www.health.gov.au/news/health-alerts/novel-coronavirus-2019-ncov-health-alert/ what-you-need-to-know-about-coronavirus-covid-19\#symptoms. accessed 27 March 2020.

COVID-19 Case, Current Situation, 2020.

https://www.health.gov.au/news/health-alerts/novel-coronavirus-2019-ncov-health-alert/ coronavirus-covid-19-current-situation-and-case-numbers\#in-australia. accessed 27 March 2020.

COVID-19 Double, Australian Coronavirus cases appeared to be doubling every three days, published on 16 March

2020.https://7news.com.au/lifestyle/health-wellbeing/australia-coronavirus-cases-appea r-to-be-doubling-every-three-days--c-747116. accessed 27 March 2020.

COVID-19 Restrictions, Public health (COVID -19 restrictions on gathering and movement) order 2020 - under the public health act 2010, published on 30 March 2020.https://www.legislation.nsw.gov.au/_emergency/Public\%20Health\%20(COVID-19 \%20Restrictions $\% 20$ on $\% 20$ Gathering\%20and\%20Movement)\%20Order $\% 202020$.pdf. accessed 31 March 2020.

DHHS, Social Distancing, published on 12 March 2020.

https://www.dhhs.vic.gov.au/coronavirus-covid-19-transmission-reduction-measures. accessed 27 March 2020.

DoH - Australian Government Department of Health, 2020. Social distancing, https://www.health.gov.au/resources/videos/coronavirus-video-social-distancing. accessed 27 March 2020.

VIC Govt., Guideline on non-essential closures due to coronavirus, published on 27 March 2020.https://www.vic.gov.au/coronavirusresponse. accessed 27 March 2020. 